\section{Review: continuing treatment with antidepressants reduces the rate of relapse or recurrence of depressive symptoms regardless of duration of treatment before or after randomisation}

\author{
Geddes JR, Carney SM, Davies C, et al. Relapse prevention with antidepressant drug treatment in depressive disorders: \\ a systematic review. Lancet 2003;361:653-61.
}

\begin{abstract}
QUESTION: In patients with depressive disorders who had previously responded to acute antidepressant therapy, does continuation of antidepressant therapy reduce the risk of relapse or recurrence of depressive symptoms more than placebo?
\end{abstract}

Source of funding: Wellcome Trust.

For correspondence: Professor J Geddes, University of Oxford, Oxford, UK. john.geddes@ psych.ox.ac.uk

\section{Data sources}

Studies were identified by searching Medline (1966 to April 2000); CINAHL (1982 to April 2000); EMBASE (1980 to April 2000); the Cochrane Collaboration depression, anxiety, and neurosis controlled trials register (CCDANCTR); PsycLIT (1974 to April 2000); Psyndex (1977 to December 1999); Lilacs (1982 to December 1999); and contact with authors, experts in the field, and drug companies that manufacture antidepressants.

\section{Study selection}

Studies were selected if they were randomised controlled trials (RCTs); allocated patients to continue or discontinue treatment; followed up patients for $\geq 1$ month; and included patients with depressive disorders who had either responded to treatment for an acute episode, or remained free from depressive illness for a period on antidepressants following initial response to treatment.

\section{Data extraction}

Data were extracted by 2 reviewers on patient characteristics, intervention details, and outcome measures. A third reviewer checked the data, and relapse or recurrence was counted according to the original authors' definitions. Trials were classified according to duration of treatment before randomisation (1-2 mo, 4-6 mo, or $>12 \mathrm{mo}$ ), and grouped by duration of randomised treatment $(6,12$, or $18-36 \mathrm{mo})$.

\section{Main results}

31 RCTs (4410 patients) met the selection criteria. Follow up ranged from 6-36 months. Fewer patients taking antidepressants had relapses of depressive symptoms than did those taking placebo (table), an effect that persisted for up to 36 months. In 6 studies that had data on the number of patients relapsing at $\geq 1$ year, fewer patients in the antidepressant groups had first recurrences of depressive symptoms regardless of the duration of treatment (table). However, more patients in the antidepressant groups withdrew from the study than did those in the placebo groups $(18 \% v 15 \%, \mathrm{p}=0.009)$.

\section{Conclusion}

In patients with depressive disorders who had previously responded to acute antidepressant therapy, continuation of antidepressants reduces the rate of relapse or recurrence of depressive symptoms regardless of duration of treatment before or after randomisation.
Continuation of antidepressant therapy v placebo for depressive disorder*

\begin{tabular}{|c|c|c|c|c|}
\hline \multirow[b]{2}{*}{ Outcomes } & \multicolumn{4}{|c|}{ Weighted event rates } \\
\hline & Antidepressants & Placebo & RRR (95\% Cl) & NNT (CI) \\
\hline \multicolumn{5}{|c|}{$\begin{array}{l}\text { Overall rate of relapse at } \\
6-36 \text { months ( } 32 \\
\text { studies) }\end{array}$} \\
\hline \multicolumn{5}{|c|}{$\begin{array}{l}\text { First recurrences at } \\
\text { various time intervals } \\
\text { after randomisation (6 } \\
\text { studies): }\end{array}$} \\
\hline 0-12 months & $18 \%$ & $60 \%$ & $65 \%$ (52 to 75$)$ & $3(2$ to 4$)$ \\
\hline 12-36 months & $15 \%$ & $29 \%$ & $66 \%$ (35 to 82$)$ & $8(4$ to 33$)$ \\
\hline $0-36$ months & $22 \%$ & $51 \%$ & $65 \%(54$ to 74$)$ & 4 (3 to 7$)$ \\
\hline
\end{tabular}

\section{COMMENTARY}

The studies reviewed by Geddes et al support 3 major conclusions regarding maintenance antidepressant treatment: (a) the continuation of antidepressant treatment reduced the risk of depression relapse by more than half; (b) in the current illness episode, the risk of relapse and the benefit of continued treatment did not vary according to duration of treatment; and (c) the advantage of continued treatment was consistent across studies that had widely varying overall rates of relapse.

The findings by Geddes et al support an emerging consensus that depression is often a chronic illness. Although traditional thinking distinguishes between acute/ continuation antidepressant treatment (presumed necessary in all cases) and maintenance treatment (only necessary in those at high risk of relapse), this distinction is clearly not supported by this review. For many patients, depression follows a chronic waxing and waning course. Therefore, treatment and service planning should follow models of other chronic relapsing illnesses such as diabetes or asthma.

The practising clinician in primary or specialty care could ask 2 questions regarding the applicability of these findings. Firstly, the physician might ask what types of patients were typically excluded from relapse prevention studies. To reach the point of randomisation, patients must achieve remission during acute treatment and maintain remission for several weeks. In everyday practice partial recovery is the modal outcome and stable remission of depression is relatively uncommon. One might presume that the question of discontinuing medication may only be relevant for this fortunate minority.

Secondly, the physician might ask whether it is possible to accurately identify those patients for whom a trial medication taper is appropriate. Relapse rates in patients switched to placebo were typically $30-60 \%$, which implies that $40-70 \%$ of patients fared reasonably well for 1-2 years after antidepressant discontinuation. It may be beneficial to formulate questions about long term antidepressant treatment in the same manner as other chronic psychiatric illnesses such as bipolar disorder or schizophrenia. Relevant questions could include: What clinical characteristics predict a lower risk of relapse with a trial medication discontinuation? What are the short and long term consequences of an unsuccessful medication taper? Answers to these questions would help patients and physicians assess the risks and benefits of tapering antidepressants more accurately. Gregory Simon, MD, MPH

Center for Health Studies/Behavioral Health Service, Seattle, Washington, USA 